\title{
P-bRS: A Physarum-Based Routing Scheme for Wireless Sensor Networks
}

\author{
Mingchuan Zhang, Wangyang Wei, Ruijuan Zheng, and Qingtao Wu \\ Information Engineering College, Henan University of Science and Technology, Luoyang 471023, China \\ Correspondence should be addressed to Mingchuan Zhang; zmc@bupt.edu.cn
}

Received 24 August 2013; Accepted 17 November 2013; Published 5 February 2014

Academic Editors: Y. Lu, J. Shu, and F. Yu

Copyright ( 2014 Mingchuan Zhang et al. This is an open access article distributed under the Creative Commons Attribution License, which permits unrestricted use, distribution, and reproduction in any medium, provided the original work is properly cited.

\begin{abstract}
Routing in wireless sensor networks (WSNs) is an extremely challenging issue due to the features of WSNs. Inspired by the large and single-celled amoeboid organism, slime mold Physarum polycephalum, we establish a novel selecting next hop model (SNH). Based on this model, we present a novel Physarum-based routing scheme (P-bRS) for WSNs to balance routing efficiency and energy equilibrium. In P-bRS, a sensor node can choose the proper next hop by using SNH which comprehensively considers the distance, energy residue, and location of the next hop. The simulation results show how P-bRS can achieve the effective trade-off between routing efficiency and energy equilibrium compared to two famous algorithms.
\end{abstract}

\section{Introduction}

Wireless sensor networks (WSNs) are a class of wireless ad hoc networks which consist of a set of sensor nodes and aim at several applications, such as industrial sensing and control and environment monitoring [1]. Each sensor node is a lowcost, short range wireless transceiver typically equipped with a low-computation processor and a battery operated power supply. Under many cases, sensors need to work without battery replacement for several years. Thus, there are two questions needing to be considered. One is how to achieve the energy balance of sensor nodes to avoid the emergence of energy hole which commonly occurs around the Sink, since the data traffic follows a many-to-one communication pattern and nodes nearer the Sink have to take heavier traffic load. The other is how to obtain high routing efficiency under multihop transmission circumstance, since WSNs can contain hundreds of such low-cost sensor nodes. Therefore, designing such networks should primarily focus on both routing efficiency and energy equilibrium in terms of tradeoff.

Location-aware routing protocols seem to possess high routing efficiency. However, there are two extremes in location-aware routing, the greedy strategy and the robust strategy. Greedy strategies may suffer failures to route packets to destination, while robust strategies need very high flooding rates to ensure reliability and rapid delivery of data. Thus, many location-aware routing protocols are mostly to propose methods to overcome the mentioned drawbacks. GPSR [2] is a famous greedy routing protocol, which makes greedy forwarding decisions using only information about a router's immediate neighbors. Sivrikaya et al. [3] propose randomized routing based on Markov chains to balance the load and routing performance. Kuhn et al. [4] utilize face (or perimeter) routing to go around voids in the topology. Bai et al. [5] present a routing algorithm which routes the connections in a manner that link failure does not shut down the entire stream but allows a continuing flow for a significant portion of the traffic along multiple paths to address the issues of reliability and energy efficiency. Trajcevski et al. [6] present heuristic approaches to relieve some of the routing load of the boundary nodes of energy holes in location-aware WSNs. Wang and Syue [7] propose a relay selection protocol based on geographical information, in which multihop transmission is realized by concatenation of single cluster-to-cluster hops.

The energy-aware routing attracts more attention of researcher than location-aware routing for the significance of energy. For maximizing the network lifetime, Rao and Fapojuwo [8] present a battery-aware distributed clustering and routing protocol which incorporates the state of the battery's 
remaining charge and health parameters in computing the charge utility metric at each cluster formation round. Trajcevski et al. [9] construct a data aggregation tree that minimizes the total energy cost of data transmission. By allowing the battery to rest for certain duration, without being subjected to heavy loads, Chau et al. [10] consider that a portion of the lost charge can be recovered due to the battery's recovery effect and present a battery model. A battery-aware power allocation model was studied in [11] for a single-hop transmission scheme to balance the network energy consumption based on the nonlinear battery parameters proposed in [12].

In recent years, bio-inspired technology has been concerned by researchers [13-15]. We draw the inspiration from the slime mold Physarum polycephalum which is a large and single-celled amoeboid organism. Nakagaki et al. [16] validate physarum which is apparently able to solve shortest path problems by constructing a maze. Tero et al. [17] use physarum forms of a network with comparable efficiency, fault tolerance, and cost to those of Tokyo rail system. Tero et al. [18] propose a mathematical model for the behavior of physarum and argue extensively that the model is perfect. We migrate the physarum foraging model to wireless networks to develop physarum-based routing algorithms through dimensionless analogy analysis $[19,20]$.

Based on our prior works [19-23], this paper focuses on how to choose the proper next hops to transmit data to the Sink in thinking of both routing efficiency and energy equilibrium, which is partially same to GEAR [24], other than only depending on the remaining energy [25]. The rest of this paper is organized as follows. Section 2 formulates the bioinspired model. Section 3 proposes the P-bRS routing. Section 4 evaluates our P-bRS by simulations. Finally, the conclusion is presented.

\section{System Models}

2.1. Typical WSNs Scenario. This paper considers large multihop WSNs which consist of $n$ static sensor nodes and a mobile Sink node, and the sensor nodes are distributed uniformly in a two-dimensional space. We assume that (1) all sensor nodes are aware of their locations, which may be achieved through GPS receivers or other methods; (2) each sensor node is aware of its energy residue; and (3) the Sink node moves along a certain orbit in the field and broadcasts periodically its current positions.

In WSNs, each node $i$ has a fixed circular transmission range $r$ which determines the set of sensors in which each node can communicate with node $i$ in one hop. We abstract such WSNs using a graph $G=(V, E)$, where each node $v \in$ $V$ represents a sensor and each edge $e \in E$ represents the existence of one-hop wireless link between two sensors. An example of WSNs' topology is shown in Figure 1.

The transmission range of $s$ is drawn as a dashed circle whose radius is $r$ and center is $s$. We call the angle $\theta$ to be the angle of deviation, which represents a measurement of the next hop deviating from the Sink $d$. The Euclidean distance of

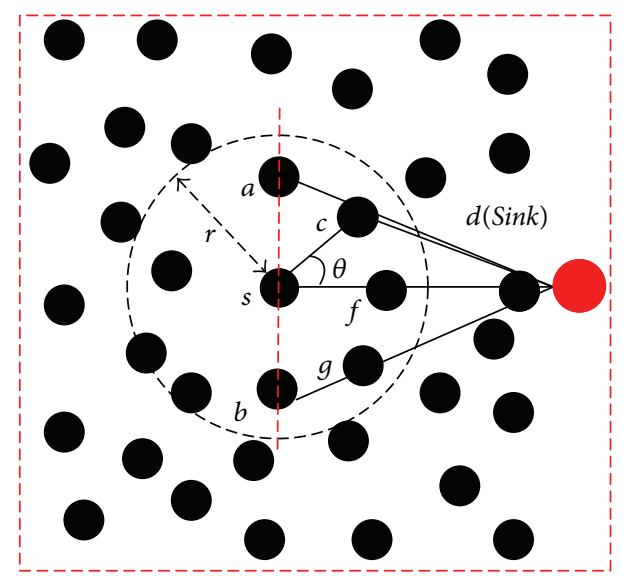

FIgURE 1: An example of WSNs' topology.

any two nodes and the angle $\theta$ can be calculated following (1) and (2), respectively. Consider

$$
\begin{aligned}
L_{i j} & =\sqrt{\left(x_{i}-x_{j}\right)^{2}+\left(y_{i}-y_{j}\right)^{2}}, \\
\theta & =\arccos \frac{L_{s c}^{2}+L_{s d}^{2}-L_{c d}^{2}}{2 \times L_{s c} \times L_{s d}},
\end{aligned}
$$

where $\left(x_{i}, y_{i}\right)$ and $\left(x_{j}, y_{j}\right)$ are the coordinates of nodes $i$ and $j$, respectively.

If the node $s$ needs to transmit data to the Sink $d$, it will select its next hop in the dashed circle. However, the nodes in semicircle far from the Sink are apparently inappropriate to be chosen as the next hop. Usually, we choose the next hop in the semicircle nearer to the Sink. Obviously, the smaller the angle $\theta$ is, the closer the next hop is to the Sink. Therefore, we are apt to choose the node whose $\theta$ is smaller as the next hop. In order to simplify discussion, we define the $N_{s}, N_{s}^{F}$, and $N_{s}^{N}$ as the sets of neighbors (the nodes in the dash circle in Figure 1), far neighbors (the nodes in the left dash circle in Figure 1), and near neighbors (the nodes in the right dash circle in Figure 1), of node $s$, respectively, where $N_{s}, N_{s}^{F}$, and $N_{s}^{N}$ satisfy $N_{s}=N_{s}^{F} \cup N_{s}^{N}$ and $N_{s}^{L} \cap N_{s}^{R}=\emptyset$.

In addition, acquiring energy residues of neighbors is important for choosing next hop to balance the energy of sensor's nodes. We think of the basic theory of wireless transmission combing with Figure 1. If node $s$ transmits a group of data to $c$, all of the nodes in $N_{s}$ would receive the wireless radio and check the packet header. The node $c$ matches the field Destination and receives the packet. Other nodes mismatch the field Destination, then ignore the packet, and go on sleeping.

In order to acquire the energy residue of neighbors, we add a new field Energy_Residue to the packet header. When node $s$ transmits a group of data to $c$, all of the nodes in $N_{s}$ extract the fields of Source and Energy_Residue from the packet header and save Energy_Residue in local memory according to field Source. Then, the node $c$ matches the field Destination and receives the packet. Other nodes mismatch the Destination, then ignore the packet, and go on sleeping. 


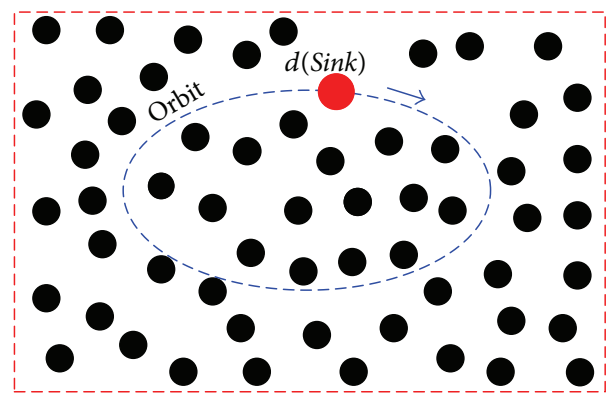

Figure 2: The orbit of the Sink node.

Since each node needs to listen in real time to every packet and try to match its field Destination, only adding an operation of saving Energy_Residue would not add a considerable effect on energy consumption. Therefore, we neglect the cost of acquiring energy residue of neighbors.

When the Sink moves along the certain orbit, it broadcasts periodically its current positions, as shown in Figure 2. Thus, the sensor nodes can achieve the current position of the Sink in real time. When a sensor node needs to send data to the $\operatorname{Sink}$, it can calculate the $\theta$ following by the mode in Figure 1.

2.2. Selecting Next Hop Model Based on Physarum. In this section, we draw a selecting next hop model (SNH) based on physarum foraging mechanism. From paper [18], the flux through each plasmodial tube is as follows:

$$
Q_{i j}=\frac{\pi r_{i j}^{4}\left(P_{i}-P_{j}\right)}{8 \eta L_{i j}}=\frac{D_{i j}\left(P_{i}-P_{j}\right)}{L_{i j}}=\frac{D_{i j} \Delta P_{i j}}{L_{i j}},
$$

where $\Delta P_{i j}=P_{i}-P_{j}$ is the difference of pressures, $\eta$ is the viscosity of the fluid, and $D_{i j}=\pi r_{i j}^{4} / 8 \eta$ is a measure of the conductivity of the tube.

Physarum forages for distributed food sources through adapting the adaptive behavior of the plasmodium. Consider

$$
\frac{d}{d t} D_{i j}=\varphi\left(\left|Q_{i j}\right|\right)-\delta D_{i j}
$$

where $\delta$ is a decay rate of the tube and $\varphi(\cdot)$ is a monotonically increasing continuous function satisfying $\varphi(0)=0$. Since (3) and (4) come from fluid dynamics and cannot be directly used in WSNs, we should discuss how to migrate (3) and (4) to WSNs.

Firstly, we discuss the replacement of physical quantities in (3). Because the $D_{i j}$ is an inherent characteristic of the tube, we replace the $D_{i j}$ by an inherent physical quantity of wireless link-link quality $\Phi_{i j}$. Since the meaning of $L_{i j}$ is the same as that in fluid dynamics, its meaning remains in our model. The replacement of $\Delta P_{i j}$ is rather complex. If flux (e.g., fluid or data packet) wishes migrate from source to destination passing by two other nodes, the fluid tends to flow through the node with lower pressure in fluid dynamics, while data packet should be relayed by the node with higher energy residue and lower angle of deviation. Therefore, we replace the $P_{j}$ of node

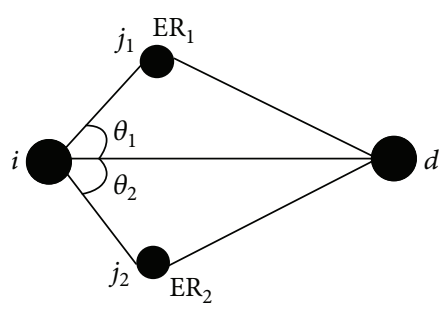

(a)

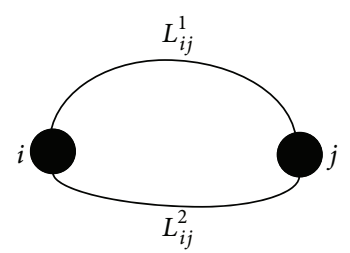

(b)
FIGURE 3: (a) If there are two candidates for next hop, the node who has the greater $k \times \mathrm{ER}+(1-k) \cos \theta$ will be picked as the next hop. (b) If two food sources are connected by two tubes, the longer tube $L_{i j}^{1}$ will vanish with time going by.

$j$ by $k \cdot \mathrm{ER}_{j}+(1-k) \cos \theta_{j i d}$, so does $P_{i}$ of node $i$. Since $P_{i}$ is the base pressure, we replace $\Delta P_{i j}$ by $P_{j}$ through omitting $P_{i}$. Using (3), we have

$$
Q_{i j}=\frac{D_{i j} \cdot \Delta P}{L_{i j}}=\frac{\Phi_{i j}\left[k \cdot \mathrm{ER}_{j}+(1-k) \cos \theta_{j i d}\right]}{L_{i j}},
$$

where $Q_{i j}$ is the virtual flux of communication through the wireless link $i j ; \Phi_{i j}$ is the link quality; $\mathrm{ER}_{j}$ is the energy residue of node $j ; L_{i j}$ is the Euclidean distance of nodes $i$ and $j ; \theta_{j i d}$ is the angle of deviation and its range is $[-\pi / 2, \pi / 2] ; k$ is a proportional coefficient which is used to adjust the weight of $\mathrm{ER}_{j}$ and $\cos \theta_{\text {jid }}$.

Because of the same characteristic of each node, we suppose that the $\Phi_{i j}$ of each link is the same and ignore it to simplify discussion. Thus, we have

$$
Q_{i j}=\frac{D_{i j} \cdot \Delta P}{L_{i j}}=\frac{k \cdot \mathrm{ER}_{j}+(1-k) \cos \theta_{j i d}}{L_{i j}} .
$$

Secondly, we analyze the adaptive behavior of plasmodium referring to Figure 3(b), where two food sources are connected by two tubes. Because $\Delta P_{i j}^{1}=\Delta P_{i j}^{2}$ and $L_{i j}^{1}>L_{i j}^{2}$, the flux $Q_{i j}^{2}$ will be greater than $Q_{i j}^{1}$ from (3). Note that $L_{i j}^{1}$ and $L_{i j}^{2}$ are kept constant throughout the adaptation process in contrast to $D_{i j}$. Therefore, the adaptive behavior of plasmodium is fulfilled by the evolution of $D_{i j}(t)$. In WSNs, node $i$ chooses the next hop form candidates as shown in Figure 3(a). Because (1) $L_{i j_{1}}$ and $L_{i j_{2}}$ are kept constant, and (2) $\Delta P_{i j_{1}}$ and $\Delta P_{i j_{2}}$ are different and time-varying, we can achieve the adaptation by the evolution of $\Delta P_{i j}(t)$. Supposing that $\varphi(Q)=Q^{\mu}$, we have

$$
\frac{d}{d t} \Delta P_{i j}=\varphi(|Q|)-\delta \Delta P_{i j}=\left(\frac{\Phi_{i j} \Delta P_{i j}}{L_{i j}}\right)^{\mu}-\delta \Delta P_{i j}
$$

where $\delta$ is a decay rate of $\Delta P_{i j}$ and $\mu$ is a constant satisfying $\mu>$ 0 . We call (7) $\mathrm{SNH}$ and use it to determine the next hop in our P-bRS; namely, we choose the node with the largest $d \Delta P_{i j} / d t$ as the next hop. 


\section{P-bRS Routing Scheme}

3.1. Data Structure in P-bRS. In this section, we introduce the data which should be conserved in each node. Each node $i$ needs to conserve the following information: $L_{i j}\left(j \in N_{i}\right)$, $\theta_{j i d}\left(j \in N_{i}^{R}\right), \mathrm{ER}_{i j}\left(j \in N_{i}\right), \mathrm{ER}_{i i}$, and $\beta_{i p j}\left(j \in N_{i}^{L}\right)$, where node $p$ is the previous hop of node $i, \mathrm{ER}_{i i}$ represents the $\mathrm{ER}_{i}$ stored in node $i$, and $\mathrm{ER}_{i j}$ represents the $\mathrm{ER}_{j}$ stored in node $i$. As our WSNs are location-aware, the $L_{i j}, \theta_{j i d}$, and $\theta_{i p j}$ are easily acquired following (1) and (2). Note that the nodes in our WSNs are fixed, and we only need to calculate the $L_{i j}$ once at WSNs deployment time. For the difference between $\mathrm{ER}_{j}$ and $\cos \theta_{j i d}$, we normalize $\mathrm{ER}_{j}$ to $\widehat{\mathrm{ER}}_{j}$. Therefore, we obtain

$$
\begin{aligned}
Q_{i j}= & \frac{k \times \widehat{\mathrm{ER}}_{j}+(1-k) \cos \theta_{j i d}}{L_{i j}}, \\
\frac{d}{d t} \Delta P_{i j}= & \left(\frac{k \times \widehat{\mathrm{ER}}_{j}+(1-k) \cos \theta_{j i d}}{L_{i j}}\right)^{\mu} \\
& -\delta\left[k \times \widehat{\mathrm{ER}}_{j}+(1-k) \cos \theta_{j i d}\right] .
\end{aligned}
$$

3.2. Routing Scheme Algorithm. If node $s$ needs to send data to the $\operatorname{Sink} d$, it searches for a routing in the following method.

Step 1. Receive the positioning information of the Sink node, and then calculate each $\theta_{j i d}$ and $\beta_{i p j}$.

Step 2. Divide each node $j \in N_{s}$ into $j \in N_{s}^{F}$ or $j \in N_{s}^{N}$ based on the positioning information of the Sink node.

Step 3. Calculate each $d \Delta P_{s j} / d t\left(j \in N_{s}^{N}\right)$ following from (9), where $\theta_{j s d}, \widehat{\mathrm{ER}}_{s j}$, and $\widehat{L}_{s j}$ are acquired by node $s$ beforehand.

Step 4. Each node $j \in N_{s}^{N}$ is saved into a temporary array variable Temp in descending order by $d \Delta P_{s j} / d t$.

Step 5. The first node in Temp is picked as the next hop of the routing.

Step 6. If the next hop $a$ of node $s$ satisfies $N_{a}^{N}=\emptyset$, namely, there is an energy hole in WSNs, the node $a$ will not send $A C K$ to $s$. Then, the node $s$ will trigger a specific processing routine.

Step 7. If $\mid$ Temp $[0] \rightarrow \theta_{j s d}-$ Temp $[1] \rightarrow \theta_{j s d} \mid \geq \pi / 2$, node $s$ will choose the node Temp[1] as the next hop. Then, the regular processing routine is going on.

Step 8. Otherwise, each $d \Delta P / d t\left(j \in N_{s}^{F}\right)$ is calculated following (10) and the nodes are saved into the Temp in ascending order by $d \Delta P / d t$. Then, the first node in Temp is chosen as the next hop of the routing and the regular processing routine is going on. Consider

$$
\begin{aligned}
\frac{d}{d t} \Delta P_{i j}= & \left(\frac{k \times \widehat{\mathrm{ER}}_{j}+(1-k) \beta_{a s j}}{L_{i j}}\right)^{\mu} \\
& -\delta\left[k \times \widehat{\mathrm{ER}}_{j}+(1-k) \beta_{a s j}\right],
\end{aligned}
$$

where $\beta_{a s j}$ is the angle of line sa and line $s j$. Equation (10) indicates that it tends to choose a node which sharply deviates from the node $a$ as the next hop to avoid entering the energy hole again.

Step 9. The process is repeated, like a rolling wheel, until the Sink $d$ is found.

3.3. Algorithm Analysis. In this section, we analyze the feasibility of SNH by mathematical theoretical analysis. We study cases in which two nodes connected to the same node compete to be the next hop, as shown in Figure 3(a).

There are four nodes $i, j_{1}, j_{2}$, and Sink $d$. For simplicity, we hereafter replace $L_{i j_{1}}, L_{i j_{2}}, Q_{i j_{1}}, Q_{i j_{2}}, \Delta P_{i j_{1}}$, and $\Delta P_{i j_{2}}$ by $L_{1}$, $L_{2}, Q_{1}, Q_{2}, \Delta P_{1}$, and $\Delta P_{2}$, respectively. In multipath routing, the virtual fluxes along each path are calculated as follows:

$$
\begin{aligned}
Q_{1} & =\frac{\Delta P_{1} / L_{1}}{\Delta P_{1} / L_{1}+\Delta P_{2} / L_{2}}, \\
Q_{2} & =\frac{\Delta P_{2} / L_{2}}{\Delta P_{1} / L_{1}+\Delta P_{2} / L_{2}} .
\end{aligned}
$$

Since $Q_{1}$ and $Q_{2}$ are nonnegative, adaptation equation (7) becomes

$$
\begin{aligned}
& \frac{d}{d t}\left(\Delta P_{1}\right)=\varphi\left(Q_{1}\right)-\delta \cdot \Delta P_{1} \\
& \frac{d}{d t}\left(\Delta P_{2}\right)=\varphi\left(Q_{2}\right)-\delta \cdot \Delta P_{2}
\end{aligned}
$$

Setting $\varphi(Q)=Q^{\mu},(d / d t)\left(\Delta P_{1}\right)=0$, and $(d / d t)\left(\Delta P_{2}\right)=$ 0 , we have

$$
\begin{aligned}
& \left(\frac{\Delta P_{1} / L_{1}}{\Delta P_{1} / L_{1}+\Delta P_{2} / L_{2}}\right)^{\mu}=\delta \cdot \Delta P_{1}, \\
& \left(\frac{\Delta P_{2} / L_{2}}{\Delta P_{1} / L_{1}+\Delta P_{2} / L_{2}}\right)^{\mu}=\delta \cdot \Delta P_{2} .
\end{aligned}
$$

From (13), we obtain

$$
\Delta P_{2}=\left(\delta^{-1 / \mu}\left(\Delta P_{1}\right)^{1-1 / \mu}-\Delta P_{1}\right) \frac{L_{2}}{L_{1}} .
$$

From (13) and (14), we obtain

$$
\Delta P_{1}=\frac{1}{\delta}\left[\frac{1}{\left(1+\left(L_{1} / L_{2}\right)^{1 / 1-\mu}\right)}\right]^{\mu} .
$$




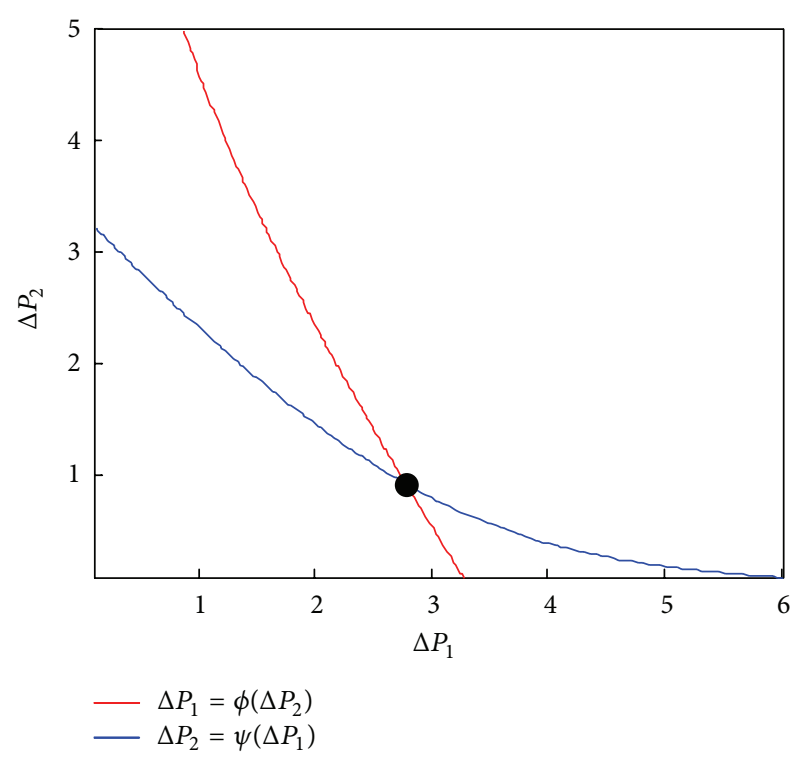

FIgURE 4: Asymptotic behavior of the solution in $\mu=0.8, \delta=0.3$, $L_{1}=10$, and $L_{2}=12$ : two curves which come from (13) intersect in a point $E$, which is the sole equilibrium point.

Similarly,

$$
\Delta P_{2}=\frac{1}{\delta}\left[\frac{1}{\left(1+\left(L_{2} / L_{1}\right)^{1 / 1-\mu}\right)}\right]^{\mu} .
$$

Namely, equilibrium point is given by $\left(\Delta P_{1}, \Delta P_{2}\right)$. We perform the simulation by setting the parameters $\mu=0.8, \delta=$ $0.3, L_{1}=10$, and $L_{2}=12$ following from (13), and the visualization of the solutions is shown in Figure 4, where point $E$ is the equilibrium point of two curves. Therefore, the routing of WSNs will reach equilibrium with our SNH, which is very important to a routing strategy.

\section{Simulation Results}

We design a simulation platform using $\mathrm{C}++$ to validate $\mathrm{P}-\mathrm{bRS}$. In the simulation, 400 sensors are relatively regularly deployed in the field of $200 \mathrm{~m} \times 200 \mathrm{~m}$, and the Sink node is deployed the right of the field, as shown in Figure 5. The sensing radius of each sensor is $30 \mathrm{~m}$, the original energy of each node is 100, and the energy of the Sink node is inexhaustible. We suppose that the energy consumption of one transmission is 1 , if the transmission distance is $20 \mathrm{~m}$. Therefore, the energy consumption of one transmission of two nodes $i$ and $j$ is $\left(L_{i j} / 20\right)$.

In order to validate the energy equilibrium, we only choose the nodes in the center or peripheral simulation field (enclosed by red dashed circle or two red dashed rectangles in Figure 5) to transmit data to the Sink. If a chosen node transmits a group of data to the Sink, the P-bRS is used to choose next hops until the Sink is found, which is called a round. This iterative process will halt after $n$ rounds until WSNs break down. We run GPSR, GEAR ( $k=0.5,0.9$, where we use $k$ to

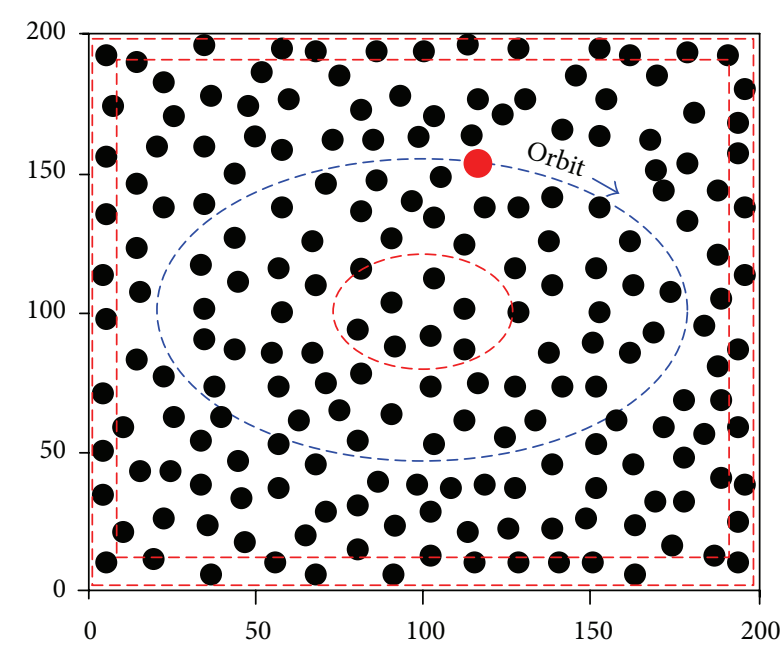

FIgURE 5: Sensors distribution.

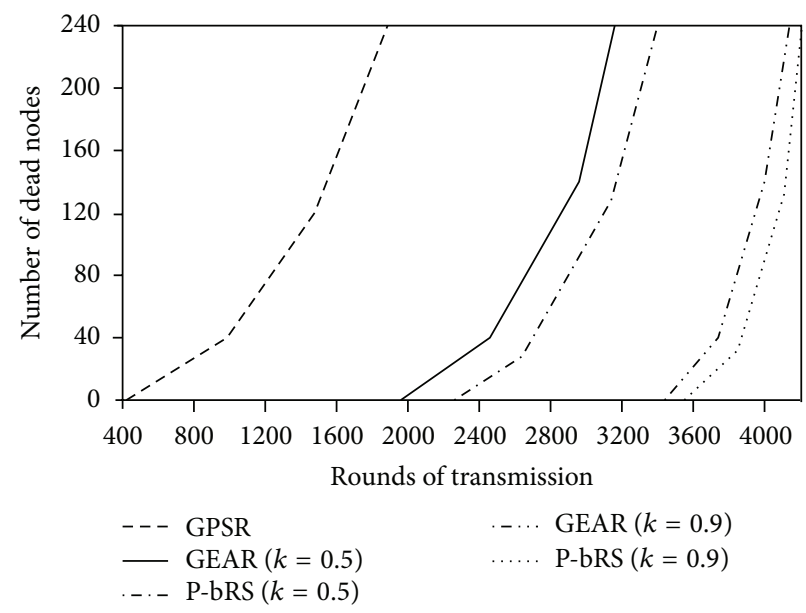

FIGURE 6: Lifetime of network.

replace $\alpha$ which is used in [24] to bring into correspondence with P-BRS $)$ and P-bRS $(k=0.5,0.9) 10$ times, respectively, to acquire their average value and compare them. If the distance between the nodes and the Sink is less than $30 \mathrm{~m}$, we set the nodes to directly transmit data to the Sink to quicken convergence of P-bRS, and the energy consumption is set to 1 .

4.1. Energy Equilibrium of P-bRS. Figure 6 illustrates the lifetime of WSNs. In GPSR, the first dead node emerges in round of 406, and the WSNs break down in round of 1857. In GEAR $(k=0.5)$, the first dead node emerges in round of 1955 , and the WSNs break down in round of 3042. In P-bRS $(k=0.5)$, the first dead node emerges in round of 2284, and the WSNs break down in round of 3351. In GEAR $(k=0.9)$, the first dead node emerges in round of 3462, and the WSNs break down in round of 4043. In P-bRS $(k=0.9)$, the first dead node emerges in round of 3524 , and the WSNs break down in round of 4108 . Therefore, the lifetime of GEAR $(k=0.5)$ is $63.8 \%$ longer than that of GPSR; the lifetime of P-bRS $(k=0.5)$ is $10.2 \%$ longer than that of GEAR $(k=0.5)$; and the lifetime of 


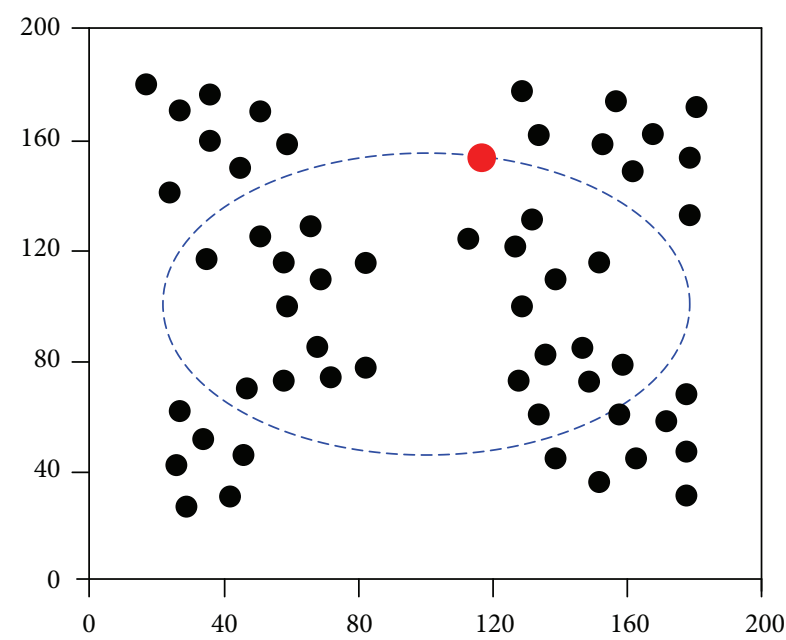

(a) P-bRS, $k=0.5$, round $=2750$

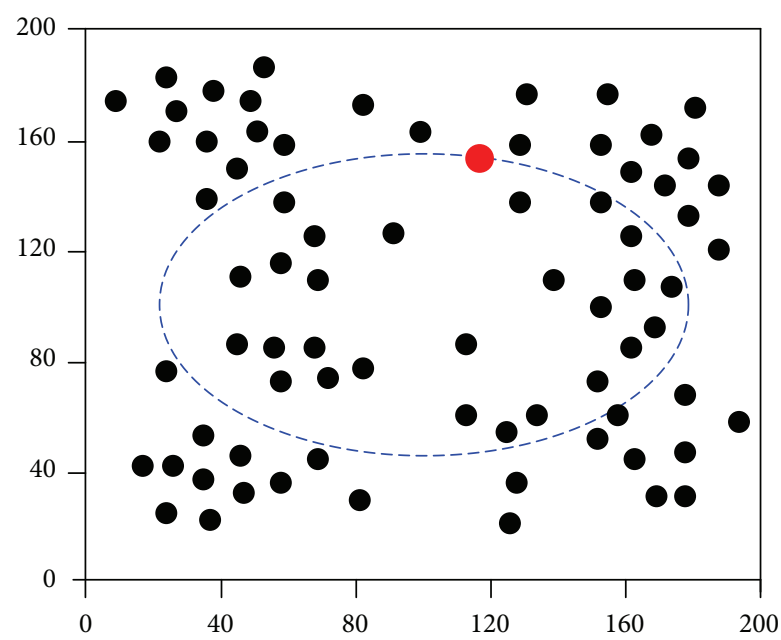

(b) GEAR, $k=0.5$, round $=2750$

FIGURE 7: Distribution of dead sensor nodes.

P-bRS $(k=0.9)$ is $1.6 \%$ longer than that of GEAR $(k=0.9)$. From Figure 6, we can differ that (1) whether considering energy residue of next hops or not will impact on the lifetime of WSNs greatly; (2) in energy balanced WSNs, the time period is very short from emerging dead nodes to networks breaking down, because all nodes reach exhausted status of energy in same time period.

Figure 7 illustrates the dead nodes distributions of GEAR $(k=0.5)$ and P-bRS $(k=0.5)$ in the rounds of 2750. The results show that P-bRS $(k=0.5)$ has much less dead nodes than GEAR $(k=0.5)$. We can also differ that the dead nodes of both algorithms are converged on a specific field but not spread around the entire range of WSNs, which is useful in deploying such WSNs to prolong the lifetime through rationally deploying the specific field.

4.2. Efficiency of P-bRS. Figure 8 illustrates the number of hops that the different algorithms need in different rounds of transmission. By calculating, the average hops of GPSR, GEAR $(k=0.5)$, P-bRS $(k=0.5)$, GEAR $(k=0.9)$, and P-bRS $(k=0.9)$, are 9.7, 12.2, 11.1, 14.8, and 13.3, respectively.

In the case of $k=0.5$, the average hop of P-bRS is $14.4 \%$ more than that of GPSR, and the hop of GEAR is $25.8 \%$ more than that of GPSR. Combing with Figure 8, the increment of average hops of $14.4 \%$ will lead to the increment of lifetime of more than $70 \%$ from GPSR to P-bRS, while the increment of average hops of $25.8 \%$ will only lead to the increment of lifetime of more than $60 \%$ from GPSR to GEAR. Therefore, the P-bRS is more efficient in balance of routing efficiency and energy equilibrium than GEAR.

In the case of $k=0.9$, the average hop of P-bRS is $37.1 \%$ more than that of GPSR, and the hop of GEAR is $52.6 \%$ more than that of GPSR. Combing with Figure 6, the increment of $14.4 \%$ of average hops will lead to the increment of about $70 \%$ of lifetime from GPSR to P-bRS $(k=0.5)$, while the increment of $19.8 \%$ of average hops will only cause the increment of $22.6 \%$ of lifetime from P-bRS $(k=0.5)$ to P-bRS $(k=0.9)$.

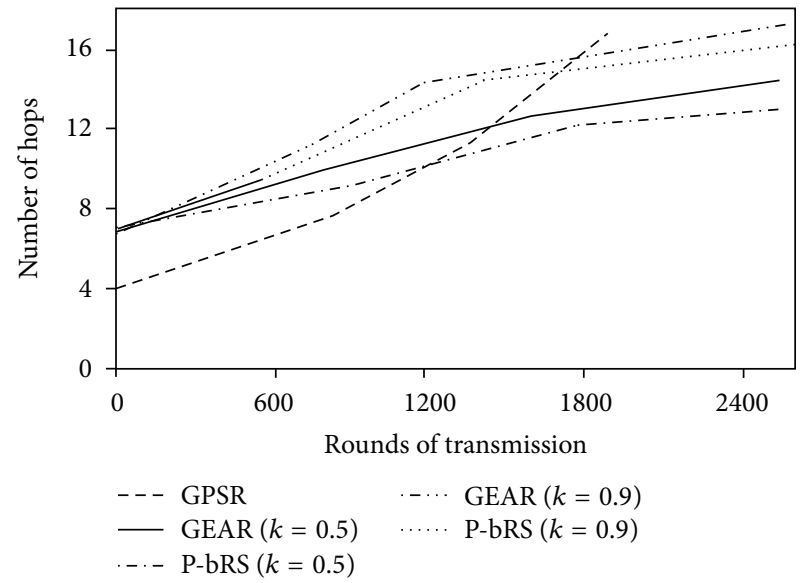

FIGURE 8: Needing hops of transmission of each round.

That is to say, the larger $k$ is, the smaller the increment of $k$ impacts on lifetime of WSNs. Therefore, it is improper to set a larger $k$, so does GEAR.

\section{Conclusion}

The physarum forages for patchily distributed food sources through accommodating its body to form networks with comparable efficiency, fault tolerance, and cost. We draw inspiration from the physarum model and improve it to suit the routing choice for WSNs. The P-bRS algorithm can deal with the trade-off between routing efficiency and energy equilibrium in WSNs, which greatly reduces the processing delay and saves the energy of sensors. Based on the simulation results, we discuss the P-bRS's performance. In future work, we consider introducing actual mobility model of nodes into P-bRS to make it fit in with mobile WSNs. Moreover, we consider the model may also provide a useful help to develop the routing protocols in other networks, which will be our future focus. 


\section{Conflict of Interests}

The authors declare that there is no conflict of interests regarding the publication of this paper.

\section{Acknowledgments}

This work is partially supported by the National Natural Science Foundation of China (NSFC), under Grant nos. U1204614, 61142002, 61003035, and 61370221, and by the key project of the Education Department Henan Province under Grant no. 14B520031, and by Key Project of Science and Technology Department of Henan Province under Grant no. 112102210187, and by the Plan for Scientific Innovation Talent of Henan Province under Grant no. 124100510006.

\section{References}

[1] B. Shuang, Z. Li, and J. Chen, "An ant-based on-demand energy route protocol for IEEE 802.15.4 Mesh network," International Journal of Wireless Information Networks, vol. 16, no. 4, pp. 225236, 2009.

[2] B. Karp and H. T. Kung, "GPSR: greedy Perimeter Stateless Routing for wireless networks," in Proceedings of the 6th Annual International Conference on Mobile Computing and Networking (MOBICOM '00), pp. 243-254, New York, NY, USA, August 2000.

[3] F. Sivrikaya, T. Geithner, C. Truong, M. A. Khan, and S. Albayrak, "Stochastic routing in wireless sensor networks," in Proceedings of the IEEE International Conference on Communications Workshops (ICC '09), Berlin, Germany, June 2009.

[4] F. Kuhn, R. Wattenhofer, and A. Zollinger, "An algorithmic approach to geographic routing in ad hoc and sensor networks," IEEE/ACM Transactions on Networking, vol. 16, no. 1, pp. 51-62, 2008.

[5] S. Bai, W. Zhang, G. Xue et al., "DEAR: delay-bounded Energyconstrained Adaptive Routing in wireless sensor networks," in Proceedings of the 31st Annual IEEE International Conference on Computer Communications (INFOCOM '12), pp. 1593-1601, Shanghai, China, March 2012.

[6] G. Trajcevski, F. Zhou, R. Tamassia, B. Avci, P. Scheuermann, and A. Khokhar, "Bypassing holes in sensor networks: load-balance vs. latency," in Proceedings of the 54th Annual IEEE Global Telecommunications Conference: "Energizing Global Communications" (GLOBECOM '11), Houston, Tex, USA, December 2011.

[7] C.-L. Wang and S.-J. Syue, "An efficient relay selection protocol for cooperative wireless sensor networks," in Proceedings of the IEEE Wireless Communications and Networking Conference (WCNC '09), Budapest, Hungary, April 2009.

[8] J. Rao and A. Fapojuwo, "A battery aware distributed clustering and routing protocol for wireless sensor networks," in Proceedings of the IEEE Wireless Communications and Networking Conference (WCNC '12), pp. 1538-1543, Shanghai, China, April 2012.

[9] G. Trajcevski, F. Zhou, R. Tamassia et al., "On the construction of data aggregation tree with minimum energy cost in wireless sensor networks: NP-completeness and approximation algorithms," in Proceedings of the Annual IEEE International Conference on Computer Communications (INFOCOM '12), pp. 25912595, Orlando, Fla, USA, March 2012.
[10] C.-K. Chau, F. Qin, S. Sayed, M. H. Wahab, and Y. Yang, "Harnessing battery recovery effect in wireless sensor networks: experiments and analysis," IEEE Journal on Selected Areas in Communications, vol. 28, no. 7, pp. 1222-1232, 2010.

[11] M. H. Chaudhary and L. Vandendorpe, "Battery-aware power allocation for lifetime maximization of wireless sensor networks," in Proceedings of the IEEE International Conference on Communications (ICC '10), Cape Town, South Africa, May 2010.

[12] J. Zhang, S. Ci, H. Sharif, and M. Alahmad, "Lifetime optimization for wireless sensor networks using the nonlinear battery current effect," in Proceedings of the IEEE International Conference on Communications (ICC '09), Dresden, Germany, June 2009.

[13] Y. Celik and E. Ulker, "An improved marriage in honey bees optimization algorithm for single objective unconstrained optimization," The Scientific World Journal, vol. 2013, Article ID 370172, 11 pages, 2013.

[14] R. Zheng, M. Zhang, Q. Wu, S. Sun, and J. Pu, "Analysis and application of bio-inspired multi-net security model," International Journal of Information Security, vol. 9, no. 1, pp. 1-17, 2010.

[15] Z. Yin, X. Liu, and Z. Wu, "A multiuser detector based on artificial bee colony algorithm for DS-UWB systems," The Scientific World Journal, vol. 2013, Article ID 547656, 8 pages, 2013.

[16] T. Nakagaki, H. Yamada, and Á. Tóth, "Maze-solving by an amoeboid organism," Nature, vol. 407, no. 6803, p. 470, 2000.

[17] A. Tero, S. Takagi, T. Saigusa et al., "Rules for biologically inspired adaptive network design," Science, vol. 327, no. 5964, pp. 439-442, 2010.

[18] A. Tero, R. Kobayashi, and T. Nakagaki, "A mathematical model for adaptive transport network in path finding by true slime mold," Journal of Theoretical Biology, vol. 244, no. 4, pp. 553564, 2007.

[19] M. Zhang, C. Xu, J. Guan, R. Zheng, Q. Wu, and H. Zhang, "PiRP: physarum-inspired routing protocol for wireless sensor networks," in Proceedings of the Vehicular Technology Conference (VTC '13), Las Vegas, Nev, USA, 2013.

[20] M. Zhang, R. Zheng, Q. Wu, and W. Wei, "A novel multi-x cooperative decision-making mechanism for cognitive internet of things," Journal of Networks, vol. 7, no. 12, pp. 2104-2111, 2012.

[21] M. Zhang, C. Xu, J. Guan, R. Zheng, Q. Wu, and H. Zhang, "A novel bio-inspired trusted routing protocol for mobile wireless 14 sensor networks," KSII Transactions on Internet and Information Systems, vol. 8, no. 1, pp. 74-90, 2014.

[22] M. Zhang, H. Zhao, R. Zheng, Q. Wu, and W. Wei, "Cognitive internet of things-concepts and application example," International Journal of Computer Science Issues, vol. 7, no. 6, pp. 151158, 2012.

[23] M.-C. Zhang, Q.-T. Wu, R.-J. Zheng, W.-Y. Wei, and G.-F. Li, "Research on grade optimization self-tuning method for system dependability based on autonomic computing," Journal of Computers, vol. 7, no. 2, pp. 333-340, 2012.

[24] Y. Yu, R. Govindan, and D. Estrin, "Geographical and Energy Aware Routing: a recursive data dissemination protocol for wireless sensor networks," UCLA Computer Science Department Technical Report UCLACSD TR-01-0023, 2001.

[25] N. Roseveare and B. Natarajan, "Energy-aware distributed tracking in wireless sensor networks," in Proceedings of the IEEE Wireless Communications and Networking Conference (WCNC '11), pp. 363-368, Cancun, Quintana Roo, Mexico, March 2011. 

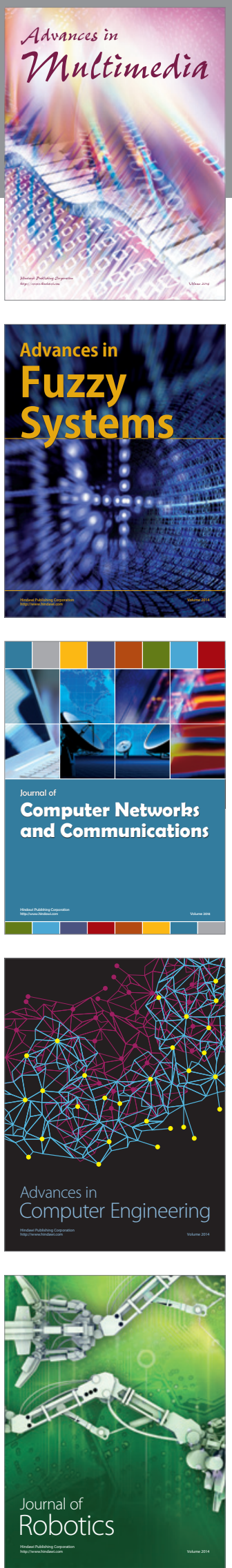

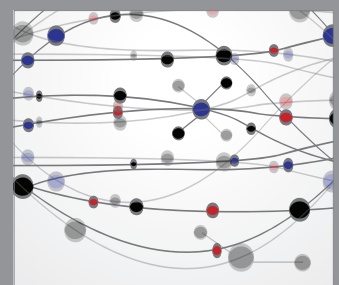

The Scientific World Journal
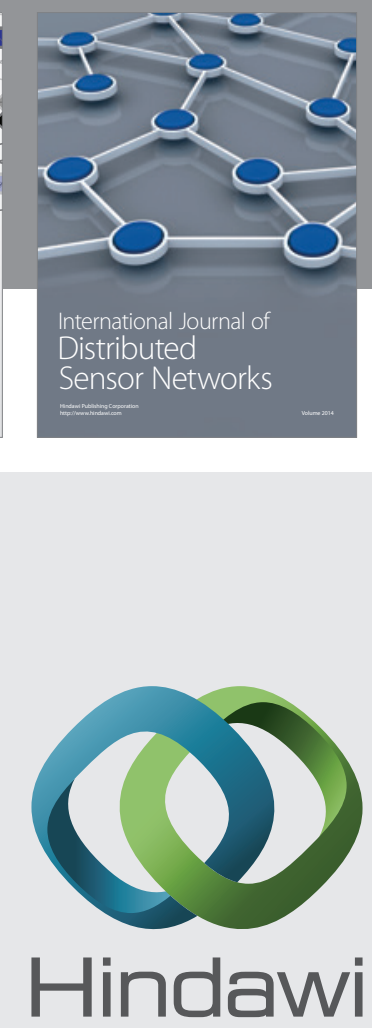

Submit your manuscripts at

http://www.hindawi.com
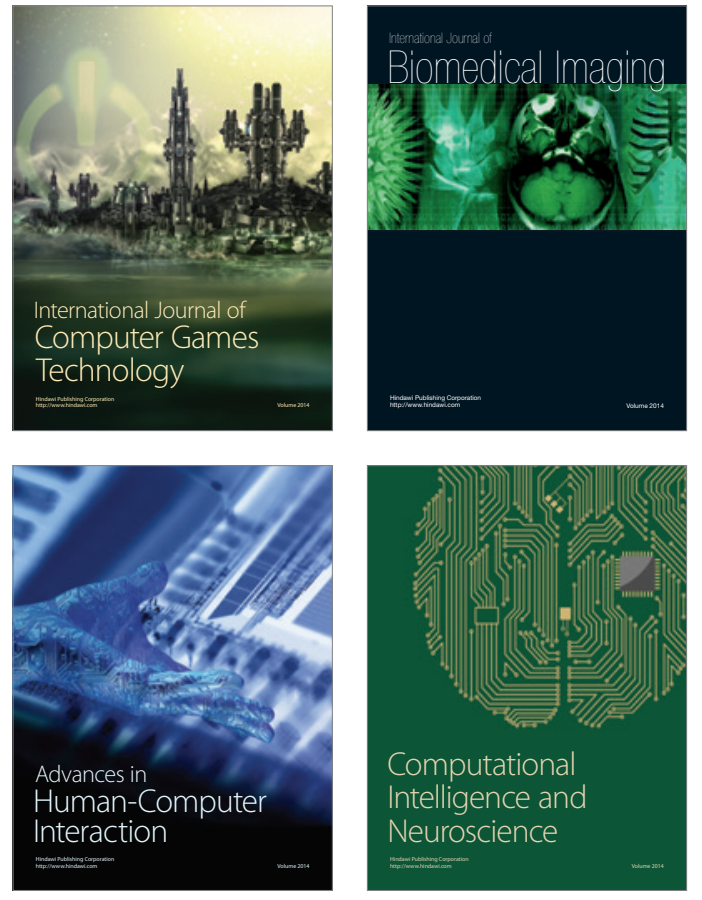
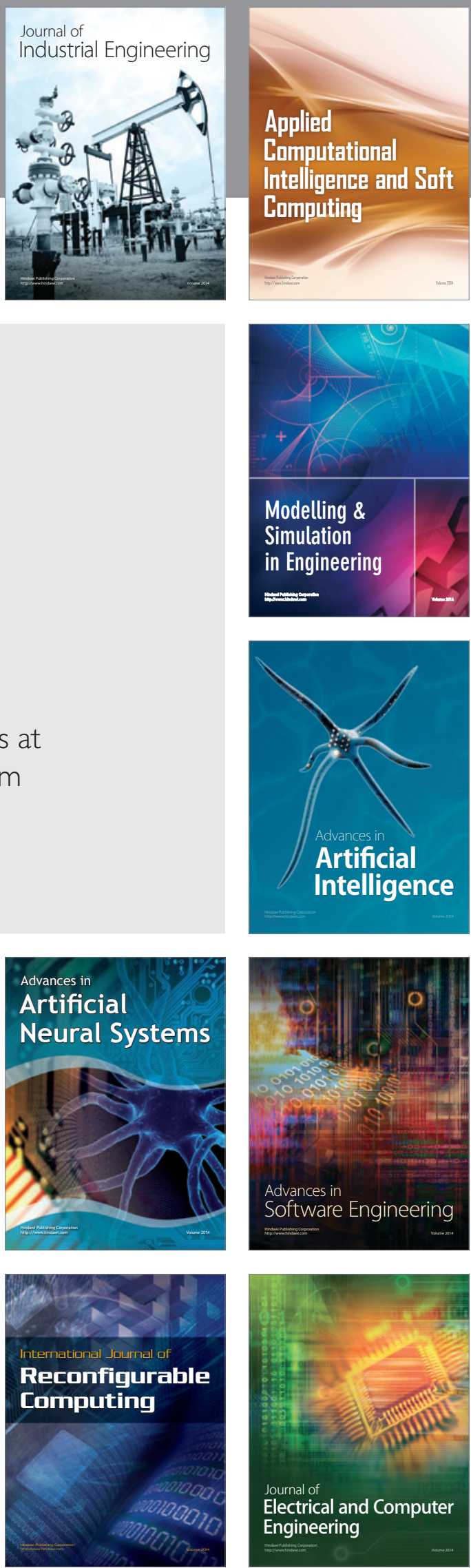